\title{
Supportive and palliative care of adults with respiratory problems experiencing structural vulnerability from homelessness, prison or other criminal justice system involvement
}

\author{
A Carole Gardener ${ }^{1}$ (orcid.org/0000-0002-8064-3780) \\ Isla Kuhn² (orcid.org/0000-0002-2879-4020) \\ Kerry Micklewright ${ }^{3}$ (orcid.org/000-0002-7559-5219) \\ Morag Farquhar ${ }^{3}$ (orcid.org/0000-0001-7991-7679)
}

${ }^{1}$ Primary Care Unit, Department of Public Health and Primary Care, University of Cambridge, Cambridge, UK

${ }^{2}$ University of Cambridge Medical School Library, University of Cambridge, Cambridge, UK

${ }^{3}$ School of Health Sciences, University of East Anglia, Norwich, UK

Corresponding author:

A. Carole Gardener

Primary Care Unit, Institute of Public Health, University of Cambridge, University Forvie Site, Robinson Way, Cambridge CB2 OSR

Tel +44 122333033

Emailacg68@medschl.cam.ac.uk 


\begin{abstract}
Purpose of review

This review seeks to identify the current prevalence of potentially life-limiting respiratory conditions among those who have experienced homelessness, incarceration or had criminal justice involvement, and current developments in, and barriers to, delivery of supportive and palliative respiratory care to these populations. These structurally vulnerable populations are known to be growing, their health behaviours more risky, and their morbidity and mortality higher, with evidence of accelerated aging.
\end{abstract}

\title{
Recent findings
}

Most studies identified investigated prevalence of respiratory conditions, which were found to be high. In contrast only one study directly explored supportive and palliative care (in a prison population) and none considered or addressed palliative and end-of-life needs of these populations, or mechanisms to address them. There was an absence of qualitative work and studies of the impact on, or role of, family, friends or informal networks.

\section{Summary}

There is a need for evidence-based interventions to reduce the risk of communicable respiratory conditions and a greater understanding of disease trajectories and management for these vulnerable populations, including provision of accessible appropriate supportive, palliative and end-of-life care.

\section{Keywords}

Respiratory, Prisons, Homelessness, Palliative 


\section{Introduction}

The health inequalities experienced by populations experiencing structural vulnerability from homelessness, prison or other criminal justice system involvement have been well documented (15).** Individuals who are homeless or in prison are more likely to experience substance misuse and poor health outcomes including high levels of long-term conditions and poor mental health. In the UK the average age of death for people experiencing homelessness is much lower than within the general population (6). Within prisons there is also evidence of 'accelerated aging' in which older prisoners are known to experience levels of morbidity and functionality normally associated with people 10 years older $(7,8)$.

Potentially life-limiting respiratory conditions such as asthma, COPD and pulmonary tuberculosis are known to be particularly prevalent in these populations suggesting a need for supportive and palliative care (9-11). However, there are unique challenges in delivering both respiratory and palliative care services to these groups $(2,5,9,12-16)$ - including the ability of individuals to access services $(9,14,16)$, delivering services that meet the particular needs of these individuals $(5,12-15)$, and their shifting demographic profiles $(2,15,17)$.

There continues to be a pressing need to understand: 1) the current prevalence and nature of potentially life-limiting respiratory conditions among those who have experienced homelessness, incarceration or who have had criminal justice involvement and 2) current developments in, and barriers to, delivery of supportive and palliative respiratory care to those experiencing these structural vulnerabilities.. A review was therefore conducted to identify, and report on recent, relevant literature.

\section{Methods}


A systematic search of the literature from 2019 to April 2020 sought to identify papers relating to supportive and palliative care of people with potentially life-limiting respiratory conditions with experience of structural vulnerability - specifically, people who have experienced homelessness (i.e. sleeping rough, living in a homeless hostel, living in insecure accommodation) or incarceration or criminal justice involvement (herein referred to as "prison populations"). The review considered full text, peer-reviewed studies of any methodology. The search strategies, run in six databases, were developed with an information specialist (IK), and are outlined in Table 1. Manual search of the reference lists of relevant papers identified through the database search checked for further potentially relevant papers based on their titles and year of publication. Two authors (CG and MF) independently screened all titles and abstracts against the inclusion and exclusion criteria and discrepancies were resolved by discussion. Full text articles of potentially relevant studies were retrieved and appraised by CG and MF for inclusion in the final review. A pragmatic approach was adopted to study appraisal in order to expedite delivery based on the five-category rating by Dixon Woods (2007) (18). Data were extracted and mapped to two emergent broad areas for both populations: (1) prevalence of potentially life-limiting respiratory conditions and (2) supportive and palliative care related to respiratory conditions.

\section{Results}

The initial search identified 287 papers in total of which 18 met the eligibility criteria and were included: six papers related to homelessness and 12 to prisons or the criminal justice system. Characteristics of included studies are summarised in Table 2. Although the search terms focused on respiratory conditions, papers rarely exclusively addressed the respiratory system (with the exception of papers relating to pulmonary tuberculosis): most addressed a broader range of health conditions, health behaviours and health care within the homeless and prison populations. By far the majority of papers related to Theme 1: prevalence of potentially life-limiting respiratory conditions within homeless and prison populations. 


\section{1) Prevalence of potentially life-limiting respiratory conditions}

Fifteen papers included data on the presence of potentially life-limiting respiratory conditions within homeless or prison populations e.g. chronic obstructive pulmonary disease (COPD), asthma, pulmonary tuberculosis (TB) or cancer. Three papers reported on tobacco use in conjunction with the prevalence of respiratory disease.

\section{Homeless populations}

Two studies reported the prevalence of non-malignant respiratory conditions among homeless people, including asthma and $\operatorname{COPD}(19,20)$. A retrospective review of medical records by Pribish et al (2019) (19) found that $65.6 \%$ of homeless people attending a free clinic $(n=183)$ reported tobacco use and $27.1 \%$ self-reported having a respiratory disease including asthma (55.2\%), COPD (41.\%), emphysema (3.4\%) or bronchitis (34.4\%). *Lewer et al (2019) (20) compared health data from a cross-sectional survey of 1,336 homeless people with that from a matched 'housed' population and found that COPD and asthma were more common in the homeless (asthma 18.3\%, COPD 14\%) than the housed group (asthma 5.7\%, COPD 1.3\%). Analysis of the prevalence of these conditions across different socio-economic groups identified 'larger and absolute differences' in prevalence of COPD in the homeless group, even in comparison to the most deprived housed socioeconomic group.

Retrospective analysis of cancer surveillance data by Holowatyi et al (2019) (21) reported that respiratory system cancers (particularly lung and bronchus) were significantly higher amongst homeless men with cancer $(n=293)$ than non-homeless men with cancer $(n=338,676)$. They identified in excess of $51 \%$ in incidence of respiratory malignancies in the homeless, increasing to in excess of $61 \%$ for lung and bronchus cancer.

Gomez et al (2019) (22) retrospectively analysed routinely collected health data to identify risk factors associated with unsuccessful treatment of TB in homeless and non-homeless populations in Columbia, both with and without HIV. Identified risk factors included homelessness, HIV 
seropositivity, male gender, age $\geq 25$, having subsidised or no health insurance, previous treatment for TB and TB diagnosis made during hospitalisation.

Brown et al (2019) (23) identified the prevalence of tobacco use and respiratory disease within 257 homeless men attending a free clinic for medical assessment and treatment: $69 \%$ were smokers, $8 \%$ were former smokers and $21 \%$ had a respiratory condition. However, the type of respiratory conditions was not reported.

In a study of a more specific group, Jutkowitz et al. (2019) (24) compared the presence of lung disease between homeless and stably-housed veterans, all of whom had been admitted to nursing homes. Cross-sectional analysis of records found that, in the three years prior to nursing home admission, homeless veterans were significantly more like to have had a diagnosis of lung disease compared to those who were stably housed $\left(A R R_{\text {homeless }}=1.08 ; 95 \% \mathrm{Cl}=1.04-1.13\right)$. Again, however, the type of lung disease was not reported.

\section{Prison populations}

Mittal et al reported on the causes of custodial deaths from a region of India (Mittal et al, 2019) (25): of 135 deaths over a 2-year period, 100 (74\%) died in prison, 33 (24\%) in mental hospital custody, and two in police custody. Among the 92 cases in which final cause of death was declared, most were due to respiratory system involvement, with TB being the leading cause. Nearly two thirds of deaths were among those aged $16-45$ years.

Five papers related specifically to the prevalence of TB in prisons or in those with a history of incarceration; each paper related to a different country (India (26), China (27), Ethiopia (28), Iran (29) and Tunisia(30)). Tong et al (2019) (27) used cluster sampling methods to gather evidence of TB prevalence in two randomly chosen prisons in middle China. World Health Organisation-defined TB screening of 3,459 prisoners identified 40 active cases: 18 were newly diagnosed and, notably, only 
seven of the 22 old TB patients accepted TB treatment. Compared with the province's and China's general population, prisoners had 1.65 (95\% Cl: 1.13,2.41) and 2.26 times (95\% Cl: 1.65,3.11) increased odds of having active TB respectively. Farhoudi et al (29) used active case finding to screen for TB-suspected prisoners (self-report in response to health care staff explanation of symptoms) in Great Tehran Prison who were then referred to a clinic for sputum testing: this method identified a 'low' prevalence of $6.5 \%(448 / 6,900)$ TB-suspected prisoners and only nine were subsequently diagnosed (0.13\%).

Three of the five TB prevalence papers also explored smoking status and other risk factors $(26,28$, 30). Dolla et al (2019) (26) found TB prevalence to be 2.5 times higher across three prisons in southern India than in the general population in the same areas, and 3.4 times higher than the national average for India. They screened 1,854 inmates aged 15 years and above incarcerated during May-September 2016 in a prison complex with modern facilities including good ventilation. Prevalence of symptoms suggestive of TB was 35\%; 16 cases were diagnosed equating to $863 / 100,000$ population (increasing to $3,226 / 100,000$ population among those aged 56 years and above) compared to $349 / 100,000$ in the general population in the same area and $256 / 100,000$ national average. Two thirds of inmates smoked.

Tsegaye Sahle et al (2019) (28) reported prevalence of bacteriologically-confirmed TB in an Ethiopian prison, but also explored associated risk factors through cross-sectional surveys of 'residents' and 'newly-admitted entrants' ( $n=13,803$; median age 26 years). They reported an 'unexpectedly low' overall prevalence of bacteriologically confirmed TB ( $0.16 \%: 0.23 \%$ in the resident population), but did not include prisoners diagnosed pre-screening and sputum sample quality and testing was poor (e.g. majority classified as saliva). The relatively low rate of current smoking in these prisoners (16.6\%) reduced the power to detect a relationship with TB, but the higher rate among residents suggested within-prison transmission. 
A case-control study conducted by Saad-Baouab et al (2019) (30) between 2010 and 2016 in two Tunisian pulmonology departments investigated whether incarceration changed the profile of pulmonary TB. Cases $(n=58)$ were male patients hospitalised for pulmonary TB with a history of incarceration; three controls (no history of incarceration) were randomly selected for each case from the same department $(n=174)$. Cases were younger, had significantly worse socio-economic conditions and educational level, and higher risk factors (smoking, alcoholism, substance abuse, unprotected sex, tuberculosis contagen and viral hepatitis C).

Tobacco use among American non-elderly ( $<65$ years) adults with and without criminal justice involvement was explored by Winkelman et al (2019) (31) using the US National Survey on Drug Use and Health (NSDUH 2008-2016). Tobacco use was more than twice as common among those with criminal justice involvement and the disparity grew over time. There were significantly higher rates of COPD; this was reported as likely due to the intensity of tobacco use (substantially higher) and earlier age of initiation.

Three papers explored the prevalence of groups of conditions that included, but were not exclusively about, respiratory system conditions(32-34). Puglisi et al (2020) (32) explored cancer prevalence among adults with criminal justice involvement using 10 years of data from the US NSDUH (20082017) focusing on cancers related to substance use (e.g. smoking and alcohol) and those detectable with guideline-based screening. Individuals with criminal justice involvement had a higher ageadjusted prevalence of lung cancer (among other cancers), which the authors determined was likely explained by higher smoking rates and limited access to treatment programmes for nicotine-use disorder.

Udo (2019) (33) examined whether a history of incarceration was associated with increased risks for chronic medical conditions above and beyond effects of past adverse childhood experiences and recent stressful life events in a nationally representative sample of US adults (2012-2013 National 
Survey of Alcohol and Related Conditions III). The survey interviewed 36,309 non-institutionalised randomly selected individuals but included questions on incarceration history, self-reported chronic conditions and lifetime cigarette use. Overall, $12.4 \%$ reported a history of incarceration: a greater proportion of these individuals currently smoked, and they had a greater number of medical conditions. They also had significantly higher odds of diagnoses for the majority of chronic medical conditions, including lung problems, even when adjusting for sociodemographic and other covariables, suggesting statistical independence from prior or current stressful life experiences and possible lifelong implications of incarceration on health.

A systematic review, focused on the growing number of older people in prison, by *Munday et al (2019) (34) conducted a meta-analysis of prevalence data for 28 non-communicable diseases in over 93,000 individuals from prisons in 11 countries. In general, diseases responsible for the greatest morbidity and mortality in the general population had particularly high prevalence in this population. In terms of respiratory conditions, asthma was reported in nine studies and COPD in six; asthma prevalence ranged from $1 \%$ to $17 \%$ and COPD from $4 \%$ to $18 \%$. Disease prevalence was consistently higher in older people in prison, with COPD being eight times higher.

\section{2) Supportive and Palliative Care}

Three papers $(19,22)$ included data on service use for people with potentially life-limiting respiratory conditions, but only one explored palliative care.

\section{Homeless populations}

In the context of homelessness, only two studies reported on findings that considered the extent to which homeless people engaged with available care in relation to their respiratory condition. Pribish et al (2019) (19) noted that only $48.3 \%$ of homeless people with asthma or COPD within their study 
cohort reported receiving any current treatment. Gomez et al (2019) (22) also found low levels of engagement amongst those diagnosed with TB, despite provision of dedicated services for homeless people (e.g. a mobile unit to track people and dedicated TB homeless day centres and shelter). The study found that homeless people were less likely to complete therapy for TB primarily due to loss to follow up.

\section{Prison populations}

The one paper that explored palliative care for prisoners profiled frequency of palliative care consultations and the nature of consultation requests for inmates who died while being hospitalised. *Stephens et al's (2019) (35) retrospective chart review of 299 inmate decedents at a contracted external facility in the United States (2007-2016), found 136 (45\%) had had a palliative care consultation prior to death (median age 56 years). Consultations were primarily for goals-of-care or transitions-of-care discussions $(50 \%, n=68)$, although code status discussions were noted for less than a fifth (9\%, $\mathrm{n}=22)$. Opioids for dyspnoea or pain was the most common medication offered (112 patients: $82 \%)$. Of those who had palliative care consultations, $70.5 \%(n=96)$ were either directly admitted or later transferred to the palliative care service. Forty seven percent of decedents $(n=141)$ had a diagnosis of malignancy, mostly lung cancer (33\% of malignancies). Nearly two thirds of patients with cancer received palliative care consultations (64\%) compared with less than a third $(29 \%)$ of those without cancer $(p<0.000)$. Consultations were close to death: median 3 days predeath.

\section{Discussion}


Although there is a growing body of literature that addresses the need for, and delivery of, supportive palliative care within homeless and prison populations in general, this review has identified a paucity of focus on investigating these issues in relation to adults with respiratory conditions who experience such structural vulnerability.

Responding to the initial lack of papers identified on scoping, a pragmatic inclusive approach was adopted to screening and applying the inclusion criteria (e.g. including studies of criminal justice system involvement in addition to prison inmates). This resulted in the identification of studies that considered respiratory conditions more generally within the homeless and prison populations (not specifically in relation to supportive and palliative care), facilitating inclusion of potentially lifelimiting respiratory conditions such as TB.

The majority of identified studies investigated the prevalence of respiratory conditions (typically in conjunction with other health conditions). High levels of respiratory disease were evidenced for both populations (the homeless and prisoners) including non-malignant non-communicable conditions (e.g. COPD and asthma), respiratory-related cancers and TB. These findings are in line with results of studies published before the review period that similarly considered prevalence $(2,9)$. It is worth noting however that the review may under-report the true extent of respiratory conditions for homeless populations in particular as many studies used 'self-report' or reports from those who have already engaged with services. The evidence suggests that people who are homeless and have respiratory problems may not attribute their symptoms to chronic ill health or may not choose, or be able, to engage with services (9). Predominant conclusions of the body of papers relating to the prevalence of these conditions in prison populations centred on the need for screening programmes.

Only one paper directly considered the delivery of supportive and palliative care. As reported, *Stephens et al (2019) (35) provided a quantitative overview profiling palliative consultations and patient outcomes for prisoners accessing inpatient care externally. However, neither this nor any other study considered how supportive and palliative care was delivered in prisons themselves, or its 
effectiveness, or highlighted particular palliative and end of life issues faced by prisoners and those who support or care for them (in either clinically or custodially). Similar conclusions can be made in relation to the homelessness literature. Two studies $(19,22)$ identified low levels of engagement in respiratory treatment and disease management programmes: findings also identified in earlier work (9). However, as with prisoners, there was no exploration of issues relating specifically to supportive and palliative care. It is also noteworthy that none of the identified studies (for either homelessness or prison settings) were qualitative; this highlighs the lack of new understandings in relation to the experience of living with life-limiting respiratory conditions, or delivering, or receiving, supportive and palliative care in these contexts. Given that most of the papers identified focused on the high prevalence of these conditions (or risk factors for them e.g. tobacco use), there is a clear need for further work to understand how supportive and palliative care is being delivered to these structurally vulnerable individuals with respiratory conditions, the barriers and facilitators that currently exist, and how best to provide care and support towards the end of life.

In addition to the dearth of studies focused on health care delivery, there was a total absence of studies considering the impact on, and role of, family, friends or informal networks in the provision of supportive and palliative care in this literature. Concerns emerging around COVID-19 at the time of writing this review further highlighted the particular vulnerability of these populations to infectious respiratory life-threatening infections both during the current pandemic and in future similar epidemics and pandemics

\section{Conclusion}

In conclusion, there remains a need for evidence-based interventions to reduce the risk of communicable respiratory conditions and a greater understanding of disease trajectories and management for these structurally vulnerable populations. This includes the provision of accessible, appropriate and effective supportive, palliative and end-of-life care that addresses the needs of these individuals, and those who support them formally and informally. 


\section{Key Points (3-5)}

- Respiratory disease is highly prevalent in the homeless, prisoners or those experiencing the criminal justice system

- There is a lack of contemporary information about the nature of supportive and palliative care currently delivered to homeless and prison populations with respiratory disease, and the experience of patients, informal carers, and care providers

- There is a need for greater understanding of barriers to effective supportive and palliative care provision for these vulnerable populations and the development of evidence-based interventions to address them

\section{Acknowledgements}

MF was part funded by the National Institute of Health Research (NIHR) Applied Research Collaboration East of England (ARC EoE) programme. The views expressed are those of the authors, and not necessarily those of the NIHR, NHS or Department of Health and Social Care.

\section{Conflicts of Interest}

None

\section{References and Recommended Reading}

Papers of particular interest, published within the period of review have been highlighted as * to denote they are of special interest and ${ }^{* *}$ to denote outstanding interest 
1. Cream J, Fenney D, Williams E, Baylis A, Dahir S, Wyatt H. Delivering health and care for people who sleep rough. Going above and beyond: The Kings Fund; 2020 [Available from: https://www.kingsfund.org.uk/sites/default/files/2020-02/Delivering-health-care-people-sleeprough-summary.pdf.

2. Fazel S, Geddes JR, Kushel M. The health of homeless people in high-income countries: descriptive epidemiology, health consequences, and clinical and policy recommendations. Lancet. 2014;384(9953):1529-40.

3. Aldridge RW, Menezes D, Lewer D, Cornes M, Evans H, Blackburn RM, et al. Causes of death among homeless people: a population-based cross-sectional study of linked hospitalisation and mortality data in England. Wellcome open research. 2019;4:49.

4. Binswanger IA, Krueger PM, Steiner JF. Prevalence of chronic medical conditions among jail and prison inmates in the USA compared with the general population. Journal of Epidemiology and Community Health. 2009;63(11):912.

5. Hudson H, Wright DK. Towards a Guiding Framework for Prison Palliative Care Nursing Ethics. ANS Advances in nursing science. 2019;42(4):341-57.

6. Office for National Statistics. Deaths of homeless people in England and Wales: 2018: Office for National Statistics; 2018 [Available from:

https://www.ons.gov.uk/peoplepopulationandcommunity/birthsdeathsandmarriages/deaths/bulleti ns/deathsofhomelesspeopleinenglandandwales/2018.

7. Munday D, Leaman J, O'Moore E. Health and social care needs assessments of the older prison population. A guidance document Public Health England; 2017 [Available from:

https://assets.publishing.service.gov.uk/government/uploads/system/uploads/attachment data/file 662677/Health and social care needs assessments of the older prison population.pdf.

8. Hayes AJ, Burns A, Turnbull P, Shaw JJ. The health and social needs of older male prisoners. International Journal of Geriatric Psychiatry. 2012;27(11):1155-62.

9. Groundswell. Room to breathe 2016 [Available from: https://groundswell.org.uk/what-wedo/peer-research/room-to-breathe/.

10. Fazel S, Hope T, O'Donnell I, Piper M, Jacoby R. Health of elderly male prisoners: worse than the general population, worse than younger prisoners. Age Ageing. 2001;30(5):403-7.

11. Dara M, Chorgoliani D, De Colombani P. TB prevention and control care in prisons. 2014 [cited 15/04/2020]. In: Prisons and Health [Internet]. World Health Organisation, [cited 15/04/2020]. Available from: http://www.euro.who.int/ data/assets/pdf file/0005/249188/Prisons-andHealth.pdf.

12. Hudson BF, Flemming K, Shulman C, Candy B. Challenges to access and provision of palliative care for people who are homeless: a systematic review of qualitative research. BMC Palliative Care. 2016;15(1):96.

13. Shulman C, Hudson BF, Kennedy P, Brophy N, Stone P. Evaluation of training on palliative care for staff working within a homeless hostel. Nurse Education Today. 2018;71:135-44.

14. de Veer AJE, Stringer B, van Meijel B, Verkaik R, Francke AL. Access to palliative care for homeless people: complex lives, complex care. BMC Palliative Care. 2018;17(1):119.

15. Turner M, Peacock M. Palliative Care in UK Prisons: Practical and Emotional Challenges for Staff and Fellow Prisoners. Journal of Correctional Health Care. 2017;23(1):56-65.

16. Maschi T, Marmo S, Han J. Palliative and end-of-life care in prisons: a content analysis of the literature. International journal of prisoner health. 2014;10(3):172-97.

17. Crane $M$, Joly L. Older homeless people: increasing numbers and changing needs. Reviews in Clinical Gerontology. 2014;24(4):255-68.

18. Dixon-Woods M, Sutton A, Shaw R, Miller T, Smith J, Young B, et al. Appraising qualitative research for inclusion in systematic reviews: a quantitative and qualitative comparison of three methods. J Health Serv Res Policy. 2007;12(1):42-7. 
19. Pribish A, Khalil N, Mhaskar R, Woodard L, Mirza AS. Chronic Disease Burden of the Homeless: A Descriptive Study of Student-Run Free Clinics in Tampa, Florida. J Community Health. 2019;44(2):249-55.

20. Lewer D, Aldridge RW, Menezes D, Sawyer C, Zaninotto P, Dedicoat M, et al. Health-related quality of life and prevalence of six chronic diseases in homeless and housed people: a crosssectional study in London and Birmingham, England. BMJ Open. 2019;9(4):e025192.

21. Holowatyj AN, Heath El, Pappas LM, Ruterbusch JJ, Gorski DH, Triest JA, et al. The Epidemiology of Cancer Among Homeless Adults in Metropolitan Detroit. JNCI Cancer Spectr. 2019;3(1):pkz006-pkz.

22. Gómez LM, Paniagua-Saldarriaga LA, Richert $Q$, Keynan $Y$, Montes F, López L, et al. Homelessness and HIV: A Combination Predictive of Poor Tuberculosis Treatment Outcomes and in Need of Innovative Strategies to Improve Treatment Completion. Am J Trop Med Hyg. 2019;100(4):932-9.

23. Brown MA, Gellatley W, Hoffman A, Dowdell L, Camac A, Francois R, et al. Medical complications of homelessness: a neglected side of men's health. Intern Med J. 2019;49(4):455-60.

24. Jutkowitz E, Halladay C, McGeary J, O'Toole T, Rudolph JL. Homeless Veterans in Nursing Homes: Care for Complex Medical, Substance Use, and Social Needs. Journal of the American Geriatrics Society. 2019;67(8):1707-12.

25. Mittal D, Jasbir S, Rai G, Kumar K, Sharma RK. A Two-Year Prospective Study of Custodial Deaths from Punjab Region of India. Medico-Legal Update. 2019;19(1):757-62.

26. Dolla CK, Dhanraj B, Malaisamy M, Padma Priyadarshini C, Syeed Hissar S, Natrajan M, et al. Burden of pulmonary tuberculosis in modern prison: A cross sectional prevalence survey from south India. The Indian journal of tuberculosis. 2019;66(1):189-92.

27. Tong Y, Jiang S, Guan X, Hou S, Cai K, Tong Y, et al. Epidemic Situation of Tuberculosis in Prisons in the Central Region of China. Am J Trop Med Hyg. 2019;101(3):510-2.

28. Tsegaye Sahle E, Blumenthal J, Jain S, Sun S, Young J, Manyazewal T, et al. Bacteriologicallyconfirmed pulmonary tuberculosis in an Ethiopian prison: Prevalence from screening of entrant and resident prisoners. PLoS One. 2019;14(12):e0226160.

29. Farhoudi B, SeyedAlinaghi S, Hosseini M, Pahlaviani FG, Firouzeh MM, Shahbazi M, et al. Prevalence of Tuberculosis in a Prison in Tehran by Active Case Finding. Infectious disorders drug targets. 2019;19(2):167-70.

30. Ben Saad-Baouab S, Mejri I, Bellili H, Meghdiche ML, Daghfous H, Tritar F. Does incarceration change the profile of pulmonary tuberculosis in Tunisia? La Tunisie medicale. 2019;97(4):541-50.

31. Winkelman TNA, Vickery KD, Busch AM. Tobacco use among non-elderly adults with and without criminal justice involvement in the past year: United States, 2008-2016. Addict Sci Clin Pract. 2019;14(1):2-.

32. Puglisi LB, Winkelman TNA, Gross CP, Wang EA. Cancer Prevalence Among Adults with Criminal Justice Involvement from a National Survey. Journal of General Internal Medicine. 2020;35(3):967-8.

33. Udo T. Chronic medical conditions in U.S. adults with incarceration history. Health psychology : official journal of the Division of Health Psychology, American Psychological Association. 2019;38(3):217-25.

34. Munday D, Leaman J, O'Moore E, Plugge E. The prevalence of non-communicable disease in older people in prison: a systematic review and meta-analysis. Age Ageing. 2019;48(2):204-12.

35. Stephens SL, Cassel JB, Noreika D, Del Fabbro E. Palliative Care for Inmates in the Hospital Setting. Am J Hosp Palliat Care. 2019;36(4):321-5.

*Munday et al (2019): This review provides a comprehensive international overview of the health inequalities experienced by an aging prison population.

*Stephens et al (2019): This paper is singled out as the only one identified in this review directly addressing palliative care, in either population. 
* Lewer et al (2019): This paper illustrates the enormity of the relative difference between the prevalence of long-term respiratory conditions in the homeless and housed populations in the UK.

**Cream et al (2020): This report provides a comprehensive overview of contemporary challenges in delivering healthcare to people sleeping rough in the UK. 
Table 1: Search strategies

Medline (Ovid) search strategy

Homelessness:

1 (chronic obstructive pulmonary disease* or (obstruct\$ adj3 (pulmonary or lung\$ or airway\$ or airflow* or bronch* or respirat*)) or copd or coad or cobd or aecb or emphysema or (lung adj2 (cancer* or neoplasm* or tumo?r*)) or interstitial lung disease* or ((chronic* or short* or labo?r* or difficult*) adj3 breath*) or breathless* or dyspnoea or dyspnea).mp. or exp lung diseases, obstructive/ or exp pulmonary disease, chronic obstructive/ or exp dyspnea/ or exp emphysema/ or lung neoplasm/

2 (homeless or "on the street*" or runaway* or (street* adj (child* or person* or people* or youth*)) or vagrant* or (sleep* adj2 rough)).mp. or exp homeless persons/ or exp homeless youth/

31 and 2

Prisons, incarceration or criminal justice involvement:

1 (chronic obstructive pulmonary disease* or (obstruct\$ adj3 (pulmonary or lung\$ or airway\$ or airflow* or bronch* or respirat*)) or copd or coad or cobd or aecb or emphysema or (lung adj2 (cancer* or neoplasm* or tumo?r*)) or interstitial lung disease* or ((chronic* or short* or labo?r* or difficult*) adj3 breath*) or breathless* or dyspnoea or dyspnea).mp. or exp lung diseases, obstructive/ or exp pulmonary disease, chronic obstructive/ or exp dyspnea/ or exp emphysema/ or lung neoplasm

2 (prison* or jail* or gaol* or detention* or inmate* or offender* or felon* or incarcerat* or custody or custodial* or offend* or (correction* adj3 (facilit* or unit*)) or penitentiar* or criminal* or crime*).mp. or exp criminal/ or exp prisoners/ or exp prisons

31 and 2

Date Limiters: 2019 - April 2020

Translated for: Embase via Ovid, CINAHL via Ebsco, PsycINFO via Ebsco, Cochrane Library, Web of Science Core Collection, Scopus

Abbreviations: COPD (Chronic Obstructive Pulmonary Disease), COAD (Chronic Obstructive Airway Disease), COBD (Chronic Obstructive Bronchopulmonary Disease), AECB (Acute Exacerbation of Chronic Bronchitis) 